\title{
Perfil epidemiológico dos portadores de sífilis entre 2010 e 2018 no Estado do Paraná, Brasil
}

\section{Epidemiological profile of syphilis patients between 2010 and 2018 in Paraná State, Brazil}

Maryna Rodrigues Gonçalves ${ }^{1}$, Mayara Rodrigues Gonçalves², Fernanda Yuki Ito ${ }^{3}$, Michely Mika Hirota ${ }^{4}$, Mariana Rie Hayashida ${ }^{5}$, Nathália Nakase Mizoguti ${ }^{6}$, Acácia Maria Lourenço Francisco $\mathrm{Nasr}^{7}$

1. ORCID: https://orcid.org/0000-0003-1219-3076. Acadêmica de Medicina. Universidade Federal do Paraná, Curitiba, Paraná, Brasil. E-mail: maryna.r9@gmail.com.

2. ORCID: https://orcid.org/0000-0002-3538-525X. Acadêmica de Medicina. Universidade Federal do Paraná, Curitiba, Paraná, Brasil. E-mail: mayararg9@gmail.com

3. ORCID: https://orcid.org/0000-0002-7509-0261. Acadêmica de Medicina. Universidade Federal do Paraná, Curitiba, Paraná, Brasil. E-mail: fernandayuito@gmail.com

4. ORCID: https://orcid.org/0000-0002-9636-3177. Acadêmica de Medicina. Universidade Federal do Paraná, Curitiba, Paraná, Brasil. E-mail: hirota.michely@gmail.com

5. ORCID: https://orcid.org/0000-0001-5246-0453. Acadêmica de Medicina. Universidade Federal do Paraná, Curitiba, Paraná, Brasil. E-mail: hayashidamariana@gmail.com

6. ORCID: https://orcid.org/0000-0002-5733-2706. Acadêmica de Medicina. Universidade Federal do Paraná, Curitiba, Paraná, Brasil. E-mail: nati.mizoguti@gmail.com

7. ORCID: https://orcid.org/0000-0002-7663-6094. Médica (Vigilância Epidemiológica) e Mestre (Tocoginecologia). Secretaria de Estado da Saúde do Paraná: Curitiba, PR, BR. E-mail: acácia.nasr@sesa.pr.gov.br

CONTATO: Fernanda Yuki Ito | Endereço: Alameda prudente de morais 611/apto 302. Telefone: (41) 99995-1726 - E-mail: fernandayuito@gmail.com 
RESUMO O objetivo desse trabalho foi analisar os casos de sífilis no Estado do Paraná, e descrever o perfil epidemiológico dos infectados, entre os anos de 2010 e 2018. O presente estudo é quantitativo, descritivo e observacional, foi desenvolvido com dados secundários do Sistema Nacional de Agravos de Notificação (SINAN), com a análise das taxas de detecção da sífilis adquirida. Entre 2010 e 2018 o Paraná registrou 39.900 casos de sífilis adquirida, sendo que $58,5 \%$ ocorreram em homens e $41,5 \%$ em mulheres. A faixa etária de maior incidência foi entre $20-29$ anos (26,9\%), seguida por $30-39$ anos $(21,4 \%)$. Quanto a escolaridade, a maioria dos infectados possuía ensino fundamental $(18,5 \%)$ e médio (19\%). A macrorregião leste do Estado apresentou a maior incidência de casos $(59,28 \%$ dos casos em 2016). Devido ao aumento dos casos de sífilis adquirida no Paraná entre os anos de 2010 a 2018, conclui-se a necessidade do direcionamento de ações de prevenção e rastreio.

DESCRITORES: Sífilis. Infecções por Treponema. Epidemiologia. Doenças Sexualmente Transmissíveis.

ABSTRACT The study aimed to analyze the syphilis cases in Paraná State, and to describe the epidemiological profile of infected individuals, between 2010 and 2018. The present study is quantitative, descriptive and observational; it was developed with secondary data from the National System of Disorders notification, evaluating the detection rate of syphilis acquired. Between 2010 and 2018 Paraná registered 39,900 cases of Acquired Syphilis, being 58.5\% in males and $41.5 \%$ in females. The age group with the highest incidence was between $20-29$ years (26.9\%), followed by $30-39$ years (21.4\%). As for schooling, most of the individuals in the identified cases had elementary (18.5\%) and high school education (19\%). The eastern macro- region of the state had the highest cases incidence (59.28\% of cases in 2016). Due to the increase in syphilis cases acquired in Paraná between 2010 and 2018, it concludes the need to target prevention and screening actions.

DESCRIPTORS: Syphilis. Treponemal Infections. Epidemiology. Sexually Transmitted Diseases. 


\section{INTRODUÇÃO}

1. sífilis é uma infecção sexualmente transmissível, causada pela bactéria Treponema pallidum. A via sexual é a forma predominante de transmissão da doença, mas ela também ocorre pela via sanguínea e transplacentária ${ }^{1-2}$. Na ausência de tratamento, a doença evolui ao longo de vários anos, com significativa morbimortalidade. Suas manifestações clínicas são divididas entre diferentes fases com diferentes sintomas ${ }^{1}$.

A sífilis precoce é dividida em primária, secundária e latente precoce, enquanto a sífilis tardia em latente tardia e sífilis terciária. A sífilis primária apresenta-se classicamente com uma lesão primária indolor no local da inoculação. A lesão é descrita como uma pápula ulcerada de bordos elevados, geralmente localizada nos órgãos genitais. Devido ao caráter indolor, pode passar despercebida pelos pacientes. Sua resolução ocorre dentro de 3 a 10 semanas, mesmo na ausência de tratamento. Se não tratada, a doença progride para o estágio secundário, cerca de 4 a 8 semanas após o aparecimento da lesão primária ${ }^{1,3-4}$.

A sífilis secundária é caracterizada por lesões muco cutâneas disseminadas na pele, mucosas e linfonodos, conhecidas como condiloma lata. Tais lesões classicamente afetam as palmas das mãos e as solas dos pés, tendem a simetria e não são pruriginosas. Podem estar associadas com sintomas constitucionais inespecíficos como mal-estar, febre e linfadenopatia. Apresenta resolução espontânea, mesmo na ausência de tratamento e, se não tratada, há evolução para a fase latente da doença ${ }^{1,3-4}$.

A sífilis latente é assintomática, caracterizada por sorologia positiva para sífilis, na ausência de manifestações clínicas. É frequentemente dividida em precoce, em que a infecção ocorreu há menos de dois anos, e tardia, com mais de dois anos de infecção. Pacientes com duração desconhecida da infecção devem ser tratados como sífilis tardia1. Se não tratada, a maioria dos pacientes permanecerá no estágio latente. Aproximadamente $25 \%$ desenvolvem sequelas clínicas da sífilis terciária, podendo ter acometimento sistêmico. As principais manifestações incluem doenças neurológicas (neurossífilis), doenças cardiovasculares (cardiossífilis) e lesões gengivais ${ }^{1,3-4}$.

O diagnóstico é baseado na história clínica e exames laboratoriais. Os exames utilizados variam em relação ao estágio da doença e a apresentação clínica, são divididos em exames diretos e imunológicos ${ }^{1,5-6}$. Os métodos de detecção direta possibilitam um diagnóstico microbiológico. Podemos citar a microscopia de campo escuro, a coloração de anticorpos fluorescentes, imuno-histoquímica e testes moleculares como a Reação em Cadeia da Polimerase (PCR). Já os testes imunológicos, são classificados em testes não treponêmicos (NTTs) e testes treponêmicos (TTs), utilizados para a triagem de indivíduos assintomáticos e são os métodos mais usados para diagnóstico em indivíduos que se apresentem com sinais e sintomas sugestivos de sífilis ${ }^{5-6}$. 
A primeira linha do tratamento é a penicilina $G$ benzatina, cuja dose sofre variações conforme o estágio da doença ${ }^{3}$. As ações para prevenção e controle da sífilis incluem o uso de preservativos sexuais, deteç̧ão precoce e tratamento adequado dos infectados e de seus parceiros sexuais ${ }^{2,7}$.

Segundo estimativas da Organização Mundial da Saúde (OMS), anualmente cerca de 376 milhões de novos casos de doenças sexualmente transmissíveis curáveis são diagnosticados, entre os quais 6,5 milhões são de casos de sífilis. A prevalência global de sífilis é estimada em $0,5 \%$ para homens e mulheres, com variações regionais de 0,1 a 1,6\% $\%^{8-9}$, sendo que há maior concentração de casos em países em desenvolvimento ${ }^{3}$.

No Brasil, a sífilis adquirida é um agravo de notificação compulsória desde 2010 . Os boletins epidemiológicos do Ministério da Saúde mostram um aumento da detecção de casos de sífilis adquirida, de 59,1 casos por 100 mil habitantes em 2010, para 75,8 casos em 2018'. O Estado do Paraná, em concordância com as estatísticas nacionais, apresentou elevação na incidência de sífilis adquirida durante o período de análise, com 2 casos para 100 mil habitantes em 2010, para 42,5 casos em $2016^{10}$.

\section{MÉTODO}

Trata-se de um estudo observacional, descritivo, retrospectivo, transversal, com abordagem quantitativa desenvolvido a partir de dados fornecidos pela vigilância epidemiológica estadual, por meio de informações contidas no Sistema Nacional de Agravos de Notificação (SINAN). Foi analisado o perfil epidemiológico dos casos de sífilis adquirida notificados no Estado do Paraná, no período compreendido entre 2010 e 2018.

A população do estudo foi composta por todos os casos de sífilis adquirida notificados no SINAN, no período de 2010 a $2018(\mathrm{~N}=32.559)$. As variáveis sociodemográficas analisadas foram: faixa etária, sexo, escolaridade, e a taxa de detecção da sífilis adquirida nas macrorregiões e regionais de saúde no Estado do Paraná.

Os dados foram tabulados, analisados, descritos e discutidos conforme revisão da literatura científica atual e boletins nacionais divulgados pelo Ministério da Saúde. Estão apresentados em gráficos e expressos em frequências absolutas e relativas. Para uma análise mais específica, foram calculadas as taxas de sífilis adquirida por 100.000 habitantes entre os anos de 2010 a 2018, a partir de dados disponibilizados no SINAN.

Os aspectos éticos foram respeitados e por se tratar de dados secundários, não houve registro em Comitê de Ética e Pesquisa, com dispensa de apreciação segundo os padrões éticos da Resolução $n^{\circ} 466 / 2012$ do Conselho Nacional de Saúde e das diretrizes éticas internacionais. 


\section{RESULTADOS}

No Estado do Paraná, entre 2010 e 2018, foram notificados 32.235 casos de sífilis adquirida. Em 2010, sua taxa de detecção foi de 0,7 casos para 100.000 habitantes, com aumento gradativo nos anos seguintes. Em 2018 a detecção foi de 87,5 casos por 100.000 habitantes. O maior número de diagnósticos ocorreu em 2018, com 9.928 casos notificados (Figura 1).

Figura 1. Taxas de detecção (por 100.000 habitante) de sífilis adquirida, segundo ano de diagnóstico, Paraná, 2010 a 2018.

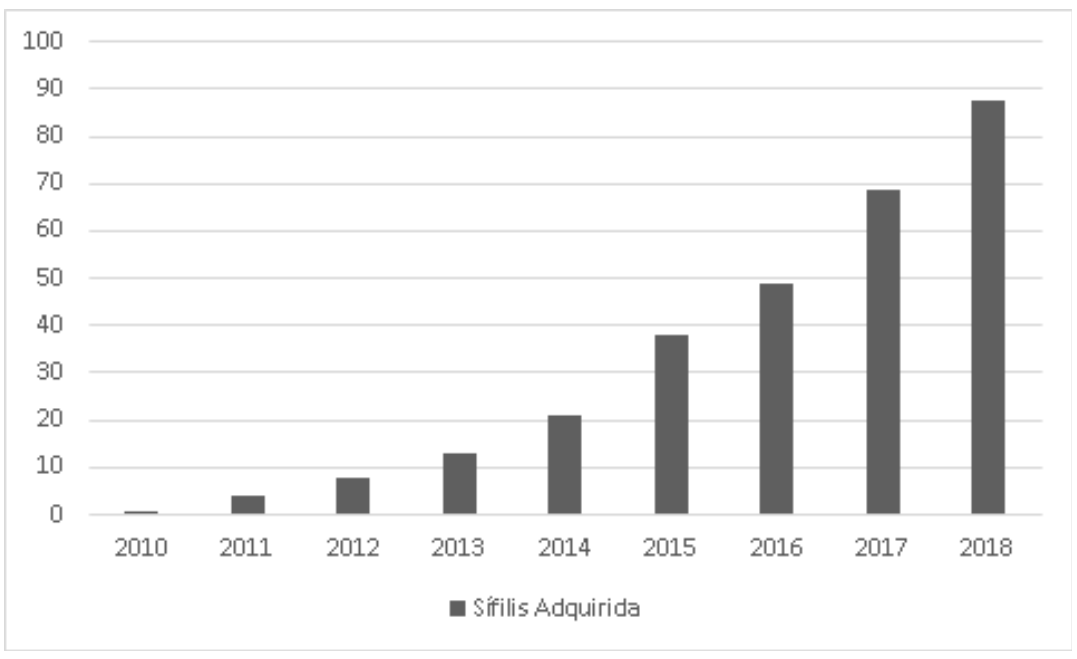

Fonte: elaborado pelos autores (2019), com dados do Boletim epidemiológico do Estado do Paraná. Secretaria da Saúde ${ }^{10}$.

No período compreendido entre 2010 e 2018 , houve mais casos notificados em homens do que em mulheres. Observa-se que dos 32.559 casos notificados, cerca de $59 \%$ ocorreram no sexo masculino é $41 \%$ no sexo feminino, com a razão de sexos de 1,4 homens para cada mulher (Figura 2).

Figura 2. Casos de sífilis adquirida segundo sexo por ano de diagnóstico. Paraná, 2010 a 2018.

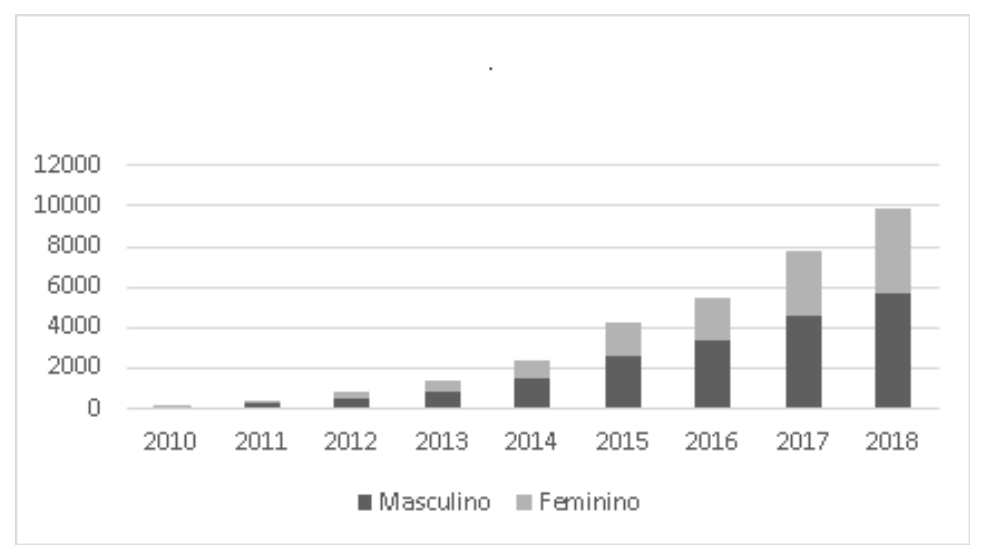

Fonte: elaborado pelos autores (2019), com dados do Boletim epidemiológico do Estado do Paraná. Secretaria da Saúde ${ }^{10}$. 
Ao analisar a distribuição por faixas etárias, a predominância no sexo masculino se mantém. No ano de 2016, a razão de sexos na faixa etária de 20 a 29 anos assume maior diferença, com a razão de 1,92 homens para cada mulher. Neste mesmo ano, a maior concentração de casos notificados, encontra-se na faixa etária de 20 a 29 anos $(33,1 \%)$, seguida pela faixa etária de 30 a 39 anos (20\%) (Figura 3).

Figura 3. Casos de sífilis adquirida por faixa etária, segundo sexo. Paraná, 2016.

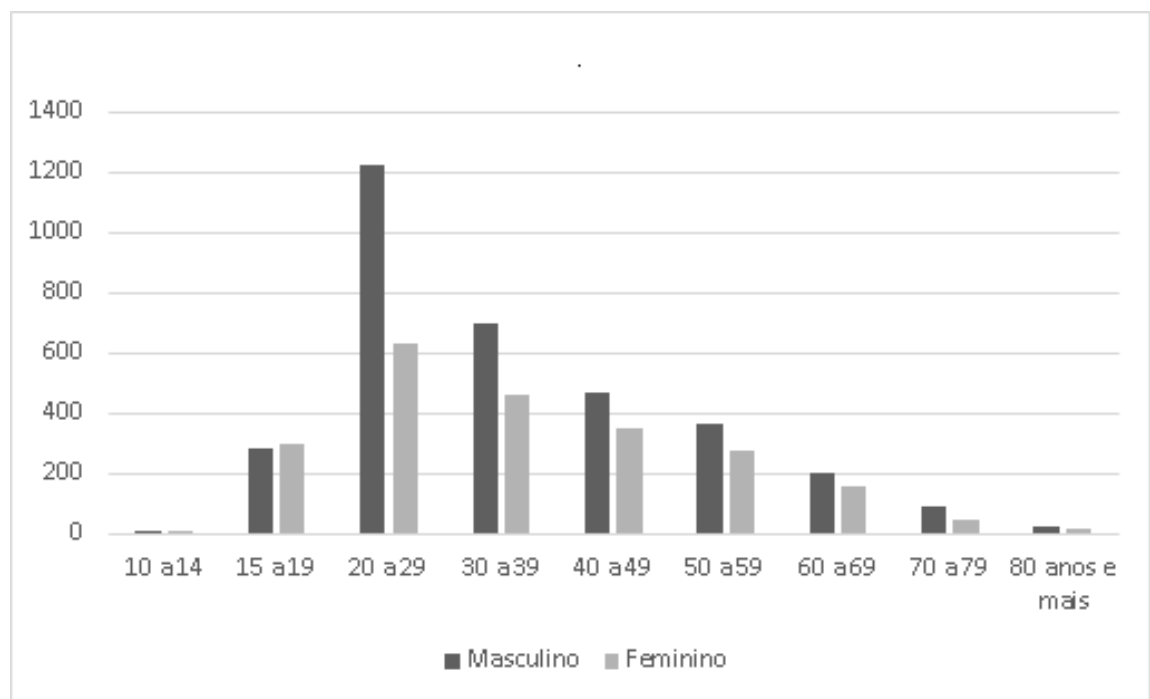

Fonte: elaborado pelos autores (2019), com dados do Boletim epidemiológico do Estado do Paraná. Secretaria da Saúde ${ }^{10}$.

Em relação à escolaridade, observa-se que os maiores percentuais de diagnóstico se encontram nas séries escolares de $5^{\mathrm{a}}$ a $8^{\mathrm{a}}$ série do ensino fundamental incompleto $(18,5 \%)$ e ensino médio completo (19\%). Ao longo dos anos, observa-se uma diminuição dos ignorados/branco, quando comparado ao ano de 2011, em que quase a metade dos casos notificados $(45,3 \%)$ foram classificados como ignorados/branco (Figura 4 ). 
Figura 4. Percentual de sífilis adquirida, segundo escolaridade. Paraná, 2010 a 2017.

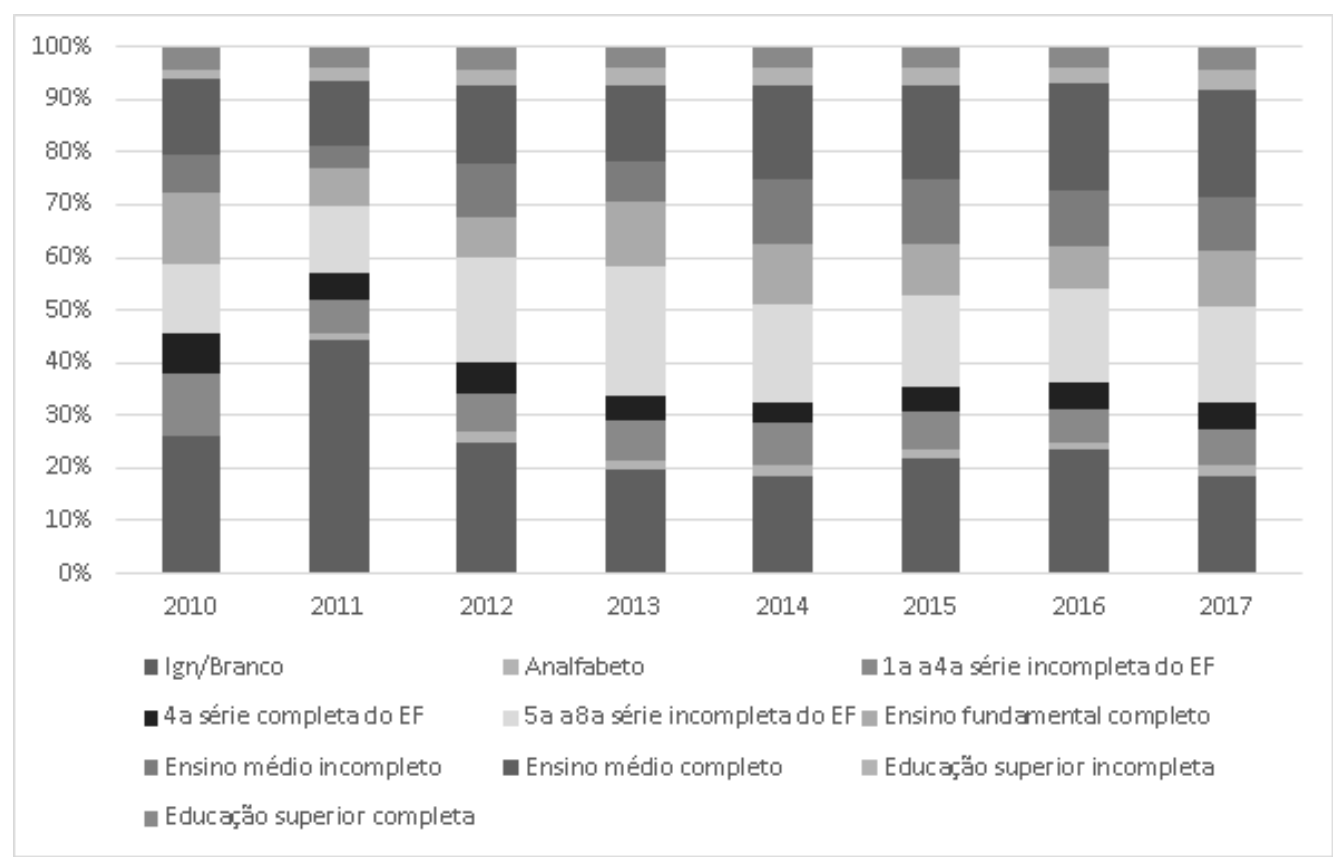

Fonte: elaborado pelos autores (2019), com dados do Boletim epidemiológico do Estado do Paraná. Secretaria da Saúde ${ }^{10}$.

Em 2016, o número total de casos notificados no Paraná foi de 5.561, com uma taxa de detecção de 49,5 casos por 100.000 habitantes. Na estratificação por macrorregiões, observaram-se $3.297(59,28 \%)$ casos notificados na macrorregião Leste, 1.258 $(22.62 \%)$ na macrorregião Oeste, 634 (11,42\%) na macrorregião Norte, 372 (6,68\%) na macrorregião Noroeste. Quanto às regionais de saúde, as taxas de detecção mais elevadas, no ano de 2016 foram observadas em Cascavel (161,9/100.000 habitantes); Metropolitana (99,4/100.000); Ponta Grossa (75,3/100.000); Pato Branco (43,8/100.000); Foz do Iguaçu (46,2/100.000); Guarapuava (96,5/100.000); Toledo (80,00/100.000); Umuarama $(53,1 / 100.000)$ e Cornélio Procópio $(41,1 / 100.000)$ (Figura 5). 
Figura 5. Taxa de deteç̧ão (por 100.000 habitantes) de sífilis adquirida, por região de saúde, segundo ano de diagnóstico. Paraná, 2016.

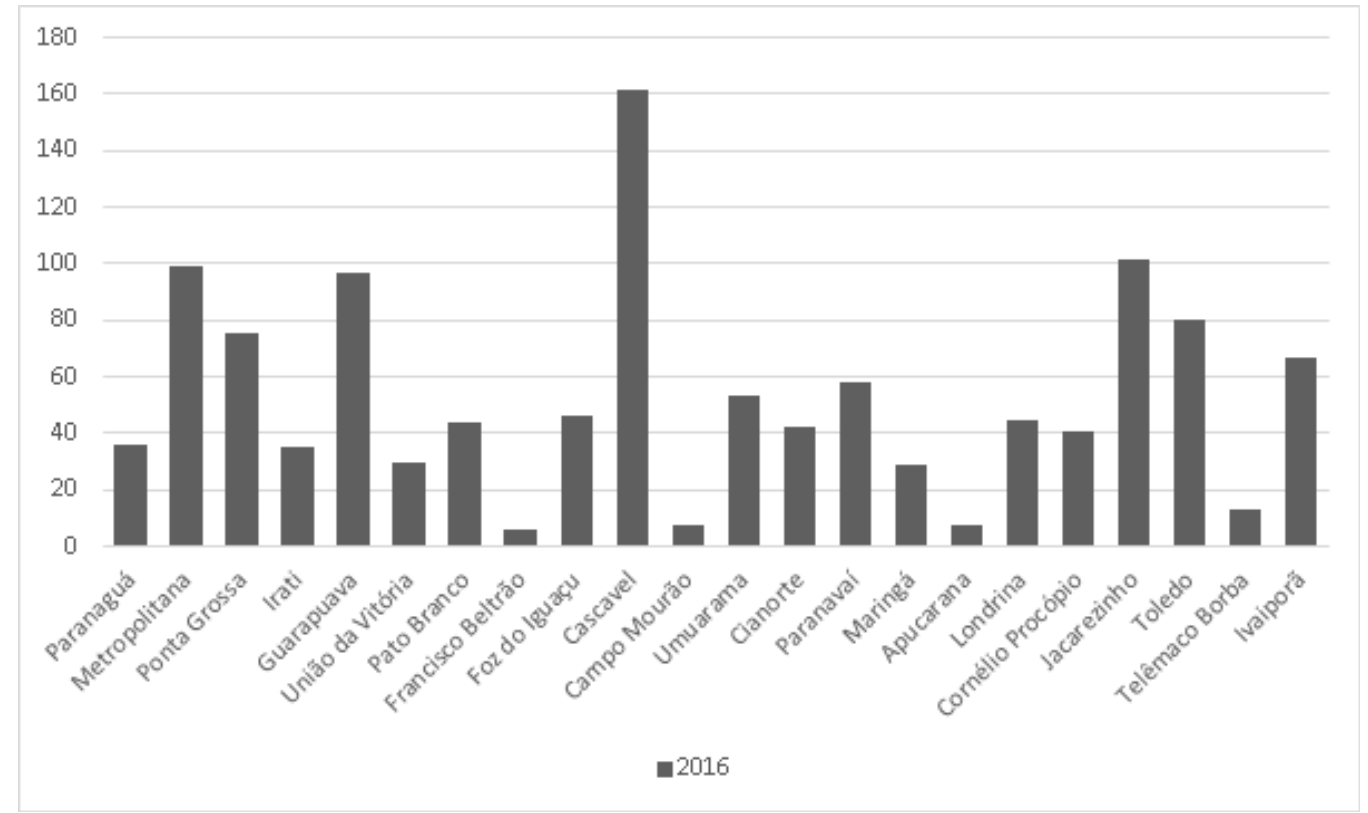

Fonte: elaborado pelos autores (2019), com dados do Boletim epidemiológico do Estado do Paraná. Secretaria da Saúde ${ }^{10}$.

\section{DISCUSSÃO}

O Brasil vive um período de aumento alarmante da transmissão de sífilis, motivo pelo qual o Ministério da Saúde incluiu a doença na lista de agravos de notificação compulsória no ano de $2010^{9,11}$. Desde então, sua taxa de detecção no território nacional e no Estado do Paraná, vem aumentando de forma significativa ${ }^{9,12}$. O aumento das notificações pode ser atribuído ao aumento do número dos casos, e também à melhoria das ações de vigilância epidemiológica, o que resulta na redução dos casos subnotificados.

Autores de um artigo nacional publicado em 2019, elaboraram três causas básicas da subnotificação dos casos de sífilis, são elas: falha nas ações de notificação e de prevenção, e desconhecimento da doença por parte dos profissionais de saúde e da população ${ }^{14}$. Outro estudo conduzido no Estado da Bahia, ressalta a elevada frequência de falhas de preenchimento das fichas de notificação, com a presença de dados ignorados ou em branco, informações ausentes ou variáveis de importância que não foram preenchidas ${ }^{15}$. Um estudo populacional conduzido no município de São Paulo, ainda cita a falta de preparo dos profissionais da saúde, que resulta em falta de orientações sobre práticas de prevenção e tratamento, e a adoção de condutas muitas vezes discrepantes com as orientações preconizadas pelo Ministério da Saúde, o que conduz à menor número de diagnósticos e aumento dos casos subnotificados ${ }^{16}$. Com o objetivo de reduzir tais falhas, é necessário reforçar a capacitação dos profissionais de saúde para o correto preenchimento das fichas de notificação e atendimento à população ${ }^{16-17}$. 
O presente estudo permitiu identificar que a notificação da sífilis adquirida no Paraná, apresentou tendência crescente no período compreendido entre 2010 e 2018. Tais dados estão em concordância com os dados nacionais divulgados pelo boletim do Ministério da Saúde ${ }^{9-10}$. Segundo o boletim, o Estado do Paraná, no ano de 2018, apresentou uma taxa de detecção de sífilis adquirida de 87,5 casos/100.000 habitantes, e esteve entre os dez estados brasileiros com taxas superiores ao da média nacional de 75,8 casos $/ 100.000$ habitantes ${ }^{10}$. Em relação às capitais, a cidade de Curitiba também apresentou uma taxa de deteç̧ão acima da média nacional, com 163,9 casos/100.000 habitantes. O presente estudo, refletiu esses dados do Ministério da Saúde, com aumento significativo dos casos de notificação conforme as regionais de saúde estaduais. A região metropolitana, compreendida na macrorregião leste do estado, apresenta a maior densidade populacional do estado $\left(193,86 \mathrm{hab} / \mathrm{km}^{2}\right)$, segundo dados populacionais do Ministério da Saúde elaborados conforme o censo 2000 do Instituto Brasileiro de Geografia Estatística $(\text { IBGE })^{10}$, e concentra a maior parte dos recursos para deteç̧ão precoce e tratamento da sífilis, resultando em maiores taxas de notificação de casos. Observamos, no entanto, que a região de Cascavel, na macrorregião oeste do estado, apesar da menor densidade populacional $\left(42,68 \mathrm{hab} / \mathrm{km}^{2}\right)$ em relação a região metropolitana, apresentou uma taxa expressiva de notificações, com 161,9 casos/100.000 habitantes. Assim como a cidade de Toledo $\left(41,78 \mathrm{hab} / \mathrm{km}^{2}\right)$, localizada também na macrorregião oeste do estado e que apresentou uma taxa de detecção de 80,00 casos/100.000 habitantes. Esse fato pode sugerir a presença de expressivo número de subnotificações na região metropolitana e em outras regionais de saúde do Estado do Paraná, como também pode ser indicativo do controle de ações de rastreamento na macrorregião oeste de saúde.

No que diz respeito ao perfil epidemiológico dos indivíduos infectados, observase que houve superioridade das notificações de sífilis adquirida em homens jovens, com idade entre 20-29 anos ${ }^{10}$. Tal achado está em acordo com a literatura atual, que aponta maior frequência de infecções de transmissão sexual em indivíduos com menos de 30 anos $^{17-18}$. Estudos populacionais apontam que esta faixa etária apresenta maior frequência de comportamentos sexuais de risco, maior número de parceiro sexuais, baixo uso de preservativos e pouco conhecimento sobre infecções sexualmente transmissíveis, ${ }^{13,17,19,20,21}$. Um estudo sobre a sífilis congênita concluiu que a maior idade é associada a menor número de parceiros sexuais, e portanto, menor risco de se adquirir a sífilis ${ }^{22}$.

Quanto às diferenças de gênero, o presente estudo também esteve em concordância com a literatura atual. Resultados de estudos populacionais mostram que quando comparado às mulheres, os homens têm início precoce da atividade sexual, maior número de parceiros/parceiras sexuais, maior consumo de drogas ilícitas e lícitas, e maior frequência de comportamentos sexuais de risco ${ }^{19,23}$. Autores de um estudo 
conduzido em São Paulo discutem os papéis de gênero na sociedade associados a maior frequência de infecções sexuais em homens, indicando que o menor uso de serviços de saúde pelos homens, maior frequência de infidelidade, sentimento de invulnerabilidade e a expressão da sexualidade masculina estão associados a comportamentos sexuais de risco ${ }^{24}$. No entanto, apesar da superioridade de notificação em homens, é válido ressaltar que a diferença na proporção entre os sexos apresenta decréscimo nos últimos anos. Resultado semelhante foi obtido em demais estudos epidemiológicos ${ }^{4,9,13}$.

O perfil de dominância das taxas no sexo masculino também é identificado em outras partes do mundo. Nos Estados Unidos, em 2018, os homens representavam 86\% dos casos notificados de sífilis no país, sendo que mais da metade desses relatavam fazer sexo com outros homens ${ }^{25}$. Um estudo conduzido na Bahia ${ }^{26}$, destacou a significativa vulnerabilidade para as doenças sexualmente transmissíveis em homens que fazem sexo com homens, associados a outros fatores de risco, como iniciação sexual precoce e a vivência em um contexto de fragilidade social. Outro trabalho ${ }^{27}$ também destacou a maior vulnerabilidade presente nos grupos de mulheres transgênero e em profissionais do sexo. Vale ressaltar que tais grupos não são alvos diretos de políticas públicas de rastreamento da sífilis, a exemplo do que ocorre com gestantes, e associado ao estigma e discriminação social resulta em falhas diagnósticas e terapêuticas.

Os resultados relativos à escolaridade estão de acordo com a literatura atual e refletem a relação entre baixa escolaridade e maior exposição a doença ${ }^{13,28-29}$. No Brasil, existe predomínio de casos de sífilis em indivíduos com ensino fundamental incompleto e ensino médio completo ${ }^{9,29}$. Uma revisão de literatura associa a baixa escolaridade a maior dificuldade de compreensão de campanhas preventivas e menor adesão ao uso de preservativos ${ }^{30}$. Estudos sobre a vulnerabilidade de adolescentes a infecções sexuais, apontam a baixa escolaridade e baixa condição socioeconômica como fator de risco ${ }^{31-33}$. A literatura atual aponta que maiores graus de escolaridade têm efeito protetor na transmissão de infecções sexuais, pois os indivíduos teriam maior preocupação em contrair essas infecções e fariam uso mais frequente de preservativos ${ }^{34-35}$. No entanto, no presente estudo, deve-se atentar sobre a falta de resposta e preenchimento inadequado da ficha de notificação neste item.

Conhecer o panorama epidemiológico da sífilis adquirida é importante para o desenvolvimento de estratégias de controle de transmissibilidade da doença, para assim prevenir a ocorrência de casos de sífilis gestacional e congênita. Nos casos de sífilis adquirida, além da notificação compulsória, as estratégias de prevenção englobam diagnóstico precoce, adesão ao tratamento tanto do paciente como de seu parceiro, uso correto de preservativos sexuais ${ }^{2,7}$. 
O presente estudo apresenta algumas limitações. Por ser um estudo retrospectivo, com o uso de dados secundários, a qualidade dos dados pode estar comprometida devido ao preenchimento inadequado das fichas de notificação. Além disso, deve-se atentar sobre a existência de casos subnotificados. Como contribuições, o estudo auxilia no conhecimento do perfil epidemiológico da sífilis no Estado do Paraná, o que pode contribuir para o desenvolvimento de ações de controle. Os resultados desta investigação apontam para a necessidade de fortalecer ações de prevenção e tratamento precoce na atenção primária de saúde, sobretudo para a parcela jovem da população.

\section{CONCLUSÃO}

Os casos de sífilis adquirida no Estado do Paraná aumentaram significativamente entre os anos de 2010 a 2018, sendo mais frequentes em jovens do sexo masculino, na região oeste e leste do estado. $O$ aumento dos casos durante os anos analisados deve levar em conta que houve uma melhora do serviço de vigilância e notificação, assim como também um aumento da cobertura dos testes rápidos.

\section{REFERÊNCIAS}

1. WHO. World Health Organization. Guidelines for the Treatment of Treponema pallidum (Syphilis). Genebra: WHO [Internet]. 2016 [acesso em 2020 Out 16]. Disponível em: https://www.ncbi.nlm.nih.gov/ books/NBK384904/

2. Luppi CG, Gomes SEC, Silva RJC, Ueno AM, Santos AMK, Tayra A, et al. Fatores associados à coinfecção por HIV em casos de sífilis adquirida notificados em um Centro de Referência de Doenças Sexualmente Transmissíveis e Aids no município de São Paulo, 2014. Epidemiol Serv Saúde [Internet]. 2018 [acesso em 2020 Outubro 16]; 27(1): e20171678. Disponível em: https://doi.org/10.5123/s167949742018000100008

3. Santos CM, Santos WM, Gallarreta FMP, Schroer CE, Edson NM. Fetal Pleural Effusion as A Echographic Marker of Congenital Syphilis. American Journal of Medical and Biological Research [Internet]. 2015 [acesso em 2020 Out 10]; 3(3): 74-75. Disponível em: http://pubs.sciepub.com/ajmbr/3/3/2/index.html

4. Bottura BR, Matuda L, Rodrigues PSS, Amaral CM, Babosa LG. Perfil epidemiológico da sífilis gestacional e congênita no Brasil - período de 2007 a 2016. Arq Med Hosp Fac Med Santa Casa São Paulo, São Paulo [Internet]. 2019 [acesso em 2020 Out 16]; 64(2): 69-75. Disponível em: http://arquivosmedicos. fcmsantacasasp.edu.br/index.php/AMSCSP/article/viewFile/515/734

5. Peeling RW, Mabey D, Kamb ML, Chen XS, Radolf JD, Benzaken AS. Syphilis. Nat Rev Dis Primers [Internet]. 2017 [acesso em 2020, Out 15]; 3: 17073. Disponível em: https://doi.org/10.1038/nrdp.2017.73.

6. Ministério da Saúde. Manual Técnico para Diagnóstico da Sífilis. Brasília, Brasil [Internet]. 2016 [acesso em 2020 Out 16]. Disponível em: http://www.aids.gov.br/pt-br/pub/2016/manual-tecnico-para-diagnosticoda-sifilis

7. Campos ALA, Araújo MAL, Melo SP, Andrade AR, Gonçalves ML. Sífilis em parturientes: aspectos relacionados ao parceiro sexual. Rev Bras Ginecol Obstet [Internet]. 2012 [acesso em 2020 Out 16]; 34(9): 397-402. Disponível em: https://doi.org/10.1590/S0100-72032012000900002

8. Rowley J, Vander S, Korenromp E, Low N, Unemo M, Abu-Raddad LJ, et al. Chlamydia, gonorrhoea, trichomoniasis and syphilis: global prevalence and incidence estimates. Bull World Health Organ [Internet]. 2019 [acesso em 2020 Out 16]; 97(8): 548-562. Disponível em: doi 10.2471/BLT.18.228486 
9. Ministério da Saúde. Secretaria de Vigilância em Saúde. Vigilância em saúde no Brasil 2003|2019: da criação da Secretaria de Vigilância em Saúde aos dias atuais. Bol Epidemiol [Internet]. 2019 [acesso em 2020 Out 15]; 50(n.esp.): 1-154. Disponível em: https://portalarquivos2.saude.gov.br/images/pdf/2019/ setembro/25/boletim-especial-21ago19-web.pdf

10. Secretaria de Estado de Saúde do Paraná. Boletim epidemiológico do Estado do Paraná, Sífilis 2018. Secretaria de Saúde [Internet]. 2018 [acesso 2020 Out 16]. Disponível em: http://www.aids.gov.br/system/ $\mathrm{tdf} /$ pub/2016/66163/boletim_sifilis_04122018.pdf?file=1\&type=node\&id=66163\&force=1

11. Ministério da Saúde, Brasil. Portaria $n^{\circ} 204$ de 17 de fevereiro de 2016. Ministério da Saúde, Gabinete do Ministro [Internet]. 2016 [acesso 2020 Out 10]. Disponível em: bvsms.saude.gov.br/bvs/saudelegis/ gm/2016/prt0204_17_02_2016.html

12. Garbin AJ, Martins RJ, Belila NM, Exaltação SM, Garbin CA. Reemerging diseases in Brazil: sociodemographic and epidemiological characteristics of syphilis and its under-reporting. Rev Da Soc Bras De Medicina Tropical. Brasilia: Soc Brasileira Medicina Tropical [Internet]. 2019 [acesso em 2020 Out 16]; 52: 4. Disponível em: doi: 10.1590/0037-8682-0226-2018

13. Souza BS, Rodrigues RM, Gomes RML. Análise epidemiológica de casos notificados de sífilis. Rev Soc Bras Clin Med [Internet]. 2018 [acesso em 2020 Out 16]; 16(2): 94-8. Disponível em: http://docs.bvsalud. org/biblioref/2018/09/913366/16294-98.pdf

14. Resplande CA, Rocha GG, Fraga GG, Gomes JMP, Santos JPV, Moura LR. Afalha na prevenção, subnotificação e conhecimento da sífilis congênita. Rev Educação em Saúde [Internet]. 2019 [acesso em 2020 Out 15]; 7(1). Disponível em: http://periodicos.unievangelica.edu.br/index.php/educacaoemsaude/article/view/3773

15. Azevedo DMS, Reis RBS, Teles MF. Incidência e Caracterização dos Casos de Sífilis Congênita na Maternidade de um Hospital do Sudoeste Baiano. Rev Mult Psic [Internet]. 2019 [acesso em 2020 Out 14]; 13(43): 387-397. Disponível em: https://idonline.emnuvens.com.br/id/article/viewFile/1542/2263

16. Saraceni V, Vellozo V, Leal MC, Hartz ZMA. Estudo de confiabilidade do SINAN a partir das Campanhas para a Eliminação da Sífilis Congênita no Município do Rio de Janeiro. Rev bras epidemiol [Internet]. 2005 [acesso em 2020 Out 12]; 8(4): 419-424. Disponível em: https://doi.org/10.1590/S1415790X2005000400010

17. Pinto VM, Basso CR, Gutierrez EB. Fatores associados às infecções sexualmente transmissíveis: inquérito populacional no município de São Paulo, Brasil. Ciênc saúde coletiva [Internet]. 2018 [acesso em 2020 Out 10]; 23(7): 2423-2432. DOI: 10.1590/1413-81232018237.20602016

18. Carret MLV, Fassa AG, Silveira DS, Bertoldi AD, Hallal PC. Sintomas de doenças sexualmente transmissíveis em adultos: prevalência e fatores de risco. Rev Saúde Pública [Internet]. 2004 [acesso 2020 Out 16]; 38(1): 76-84. Disponível em:https://doi.org/10.1590/S0034-89102004000100011

19. Taquette SR, Vilhena MM, Campos MP. Doenças sexualmente transmissíveis e gênero: um estudo transversal com adolescentes no Rio de Janeiro. Cad Saúde Pública [Internet]. 2004 [acesso em 2020 Out 16]; 20(1): 282-290. Disponível em: https://doi.org/10.1590/S0102-311X2004000100046

20. Sales WB, Caveião C, Visentin A, Mocelin D, Costa PM, Simm ED. Comportamento sexual de risco e conhecimento sobre IST/SIDA em universitários da saúde. Revista de Enfermagem Referência [Internet]. 2016 [acesso em 2020 Out 16]; IV (10): 19-27. Disponível em: http://dx.doi.org/10.12707/RIV16019

21. Benincasa M, Rezende MM, Coniaric J. Sexo desprotegido e adolescência: fatores de risco e de proteção. Psicol teor prat [Internet]. 2008 [acesso em 2020 Out 16]; 10(2): 121-134. Disponível em: http:// pepsic.bvsalud.org/scielo.php?script=sci_arttext\&pid=S1516-36872008000200010

22. Macêdo VC, Lira PIC, Frias PG, Romaguera LM, Caires SF, Ximenes RAA. Fatores de risco para sífilis em mulheres: estudo caso-controle. Rev Saúde Pública [Internet]. 2017 [acesso em 2020 Out 16]; 51:78. Disponível em: https://doi.org/10.11606/s1518-8787.2017051007066

23. Penha, JC, Aquino CBQ, Neri EAR, Reis TG, Aquino PS, Pinheiro AKB. Fatores de risco para doenças sexualmente transmissíveis em profissionais do sexo do interior piauiense. Rev Gaúcha Enferm [Internet]. 2015 [acesso em 2020 Out 16]; 36(2): 63-69. Disponível em: https://doi.org/10.1590/19831447.2015.02.52089

24. Guerriero I, José A, Hearst N. Masculinity and vulnerability to HIV among heterosexual men in São Paulo, Brazil. Rev Saúde Pública [Internet]. 2002 [acesso em 2020 Out 16]; 36(4 Supl): 50-60. Disponível em: https://doi.org/10.1590/S0034-89102002000500008 
25. Ghanem KG, Ram S. The Modern Epidemic of Syphilis. New England Journal of Medicine [Internet]. 2020 [acesso em 2020 Out 16]; 382(9): 845-854. DOI: 10.1056/NEJMra1901593

26. Brignol S, Dourado I, Amorim LD, Kerr LRFS. Vulnerability in the context of HIV and syphilis infection in a population of men who have sex with men (MSM) in Salvador, Bahia State, Brazil. Cad Saúde Pública [Internet]. 2015 [acesso em 2020 Out 16]; 31(5): 1035-1048. Disponível em: https://doi.org/10.1590/0102$311 \times 00178313$

27. Kojima N, Klausner JD. An Update on the Global Epidemiology of Syphilis. Curr Epidemiol Rep [Internet]. 2018 [acesso em 2020 Out 16]; 5(1): 24-38. Disponível em: doi: 10.1007/s40471-018-0138-z

28. Gomes NC, Rodrigues C, Meier DAP, Pieri FM, Alves E, Albanese SPR, et al. Prevalence and factors associated with syphilis in a Reference Center. Rev Soc Bras Med Trop [Internet]. 2017 [acesso em 2020 Out 16]; 50(1): 27-34. Disponivel em: https://doi.org/10.1590/0037-8682-0102-2016

29. Souza WN, Benito LAO. Perfil epidemiológico da sífilis congênita no Brasil no período de 2008 a 2014 . Universitas: Ciências da Saúde [Internet]. 2016 [acesso em 2020 Out 16]; 14(2): 1-8. Disponível: https:// doi.org/10.5102/ucs.v14i2.3811.

30. Soares JP, Oliveira AC, Silva D, Freire MEM, Nogueira JA. Prevalência e Fatores de Risco Para o HIVIAIDS em Populações Vulneráveis: Uma Revisão Integrativa de Literatura. Arq Catarin Med [Internet]. 2017 [acesso em 2020 Out 16]; 46(4): 182-194. Disponível em: http://www.acm.org.br/acm/seer/index.php/ arquivos/article/view/126

31. Mesquita JS, Costa MIF, Luna IT, Silva AA, Pinheiro PNC. Fatores de Risco e Prevenção Entre Adolescentes em Relação Às DST/HIVIAIDS. Rev enferm UFPE [Internet]. 2017 [acesso em 2020 Out 16]; 11(3): 1227-1233. DOI: 10.5205/reuol.10544-93905-1-RV.1103201713

32. Fontes MB, Campos CR, Margini SA, Duarte LD, Araújo GA, Tsuyoshi FR. Fatores determinantes de conhecimentos, atitudes e práticas em DST/Aids e hepatites virais, entre jovens de 18 a 29 anos, no Brasil. Ciência \& Saúde Coletiva [Internet]. 2017 [acesso em 2020 Out 16]; 22(4): 1343-1352. Disponível em: https://doi.org/10.1590/1413-81232017224.12852015

33. Neves RG, Wendt A, Flores TR, Costa CS, Costa FS, Rodrigues LT, et al. Simultaneidade de comportamentos de risco para infecções sexualmente transmissíveis em adolescentes brasileiros, 2012. Epidemiol Serv Saúde [Internet]. 2017 [acesso em 2020 Out 16]; 26(3): 443-454. Disponível em: http:// dx.doi.org/10.5123/s1679-49742017000300003

34. Miranda AE, Ribeiro D, Rezende EF, Pereira GFM, Pinto VM, Saraceni V. Associação de conhecimento sobre DST e grau de escolaridade entre conscritos em alistamento ao Exército Brasileiro. Brasil, 2007. Ciênc saúde coletiva [Internet]. 2013 [acesso em 2020 Out 16]; 18(2): 489-497. Disponível em: https://doi. org/10.1590/S1413-81232013000200020

35. Miranda AE, Gadelha AMJ, Szwarcwald CL. Padrão de comportamento relacionado às práticas sexuais e ao uso de drogas de adolescentes do sexo feminino residentes em Vitória, Espírito Santo, Brasil, 2002. Cad Saúde Pública [Internet]. 2005 [acesso em 2020 Out 16]; 21(1): 207-216. Disponível em: https://doi. org/10.1590/S0102-311X2005000100023

RECEBIDO: 08/06/2020

ACEITO: 08/10/2020 